\title{
On optimal approximation in periodic Besov spaces
}

\author{
Fernando Cobos ${ }^{1, *}$ \\ Departamento de Análisis Matemático y Matemática Aplicada, Facultad de Matemáticas, \\ Plaza de las Ciencias 3, 28040 Madrid. Spain. \\ Thomas Kühn ${ }^{1}$ \\ Mathematisches Institut, Universität Leipzig, Augustusplatz 10, 04109 Leipzig. Germany. \\ Winfried Sickel \\ Mathematisches Institut, Friedrich-Schiller-Universität Jena, Ernst-Abbe-Platz 2, o7737 \\ Jena. Germany.
}

\begin{abstract}
We work with spaces of periodic functions on the $d$-dimensional torus. We show that estimates for $L_{\infty}$-approximation of Sobolev functions remain valid when we replace $L_{\infty}$ by the isotropic periodic Besov space $B_{\infty, 1}^{0}$ or the periodic Besov space with dominating mixed smoothness $S_{\infty, 1}^{0} B$. For $t>1 / 2$, we also prove estimates for $L_{2}$-approximation of functions in the Besov space of dominating mixed smoothness $S_{1, \infty}^{t} B$, describing exactly the dependence of the involved constants on the dimension $d$ and the smoothness $t$.
\end{abstract}

Keywords: Approximation numbers, isotropic and mixed Besov spaces, $d$-dependence of the constants, Wiener algebra.

2010 MSC: 46E35, 41A25

\section{Introduction}

A number of problems in finance, quantum chemistry and related areas are modeled on function spaces of Sobolev type on high-dimensional domains (see, for example, [40]). For this reason there is an increasing interest nowadays in the study of linear approximation (approximation numbers) in the context of Sobolev and Besov spaces. The main point being now to determine the asymptotically optimal equivalence constants as a function of the dimension, the order of smoothness of the space and its norm.

T. Ullrich and two of the present authors have investigated in $[19,20]$ linear approximation of functions in the isotropic periodic Sobolev spaces $H^{s}\left(\mathbb{T}^{d}\right)$ and in the smaller spaces $H_{\text {mix }}^{s}\left(\mathbb{T}^{d}\right)$ of dominating mixed smoothness, determining the exact decay rate of constants as the dimension $d$ goes to infinity (see also the

\footnotetext{
* Corresponding author.

Email addresses: cobos@mat.ucm.es (Fernando Cobos), kuehn@math.uni-leipzig.de

(Thomas Kühn), winfried.sickel@uni-jena.de (Winfried Sickel)

${ }^{1}$ Supported in part by MTM2017-84058-P (AEI/FEDER, UE).
} 
paper by Dũng and Ullrich [11]). The error was measured in $L_{2}\left(\mathbb{T}^{d}\right)$. Later, the present authors [8] have studied the same problems but measuring the error in the (sometimes preferred) sup-norm. A general method was established which allows to transfer results on $L_{2}$-approximation into results on $L_{\infty}$-approximation (see [8]).

In the present paper we continue that investigation working now in the context of isotropic periodic Besov spaces $B_{p, q}^{t}\left(\mathbb{T}^{d}\right)$ and Besov spaces of dominating mixed smoothness $S_{p, q}^{t} B\left(\mathbb{T}^{d}\right)$. We show that $L_{\infty}\left(\mathbb{T}^{d}\right)$ can be replaced by any of the spaces $B_{\infty, 1}^{0}\left(\mathbb{T}^{d}\right)$ and $S_{\infty, 1}^{0} B\left(\mathbb{T}^{d}\right)$ without changing the associated approximation numbers. This result is unexpected and has some practical use, since the Littlewood-Paley characterization of the target spaces simplifies the computation of approximation or other s-numbers, see, e.g., the monographs by Dũng, Temlyakov and Ullrich [12] or by Temlyakov [35], [36]. Moreover, for $t>1 / 2$, we establish estimates for the approximation numbers of the embedding $I_{d}: S_{1, \infty}^{t} B\left(\mathbb{T}^{d}\right) \longrightarrow L_{2}\left(\mathbb{T}^{d}\right)$ which show the dependence of the involved constant on the dimension $d$ and the smoothness $s$. We also consider the approximation of tensor products of functions with finite total variation.

We start the paper by fixing the notation in Section 2. Then, in Section 3, we deal with isotropic periodic Besov spaces and, in Section 4, with Besov spaces of dominating mixed smoothness. Finally, in Section 5, we consider spaces of tensor products of functions.

\section{Preliminaries}

Let $d \in \mathbb{N}$. Given $x=\left(x_{1}, \ldots, x_{d}\right) \in \mathbb{R}^{d}$ and $0<p \leq \infty$ we put $|x|_{p}=$ $\left(\sum_{j=1}^{d}\left|x_{j}\right|^{p}\right)^{1 / p}$ if $p<\infty$, and $|x|_{\infty}=\max _{1 \leq j \leq d}\left|x_{j}\right|$ if $p=\infty$. Let $\mathbb{T}$ be the torus, i.e. $\mathbb{T}=[0,2 \pi]$ where the endpoints of the interval are identified. We put $\mathbb{T}^{d}$ for the $d$-dimensional torus. We consider on $\mathbb{T}^{d}$ the normalized Lebesgue measure $(2 \pi)^{-d} d x$. So $\left\{e^{i k x}: k \in \mathbb{Z}^{d}\right\}$ is an orthonormal basis in $L_{2}\left(\mathbb{T}^{d}\right)$, where $k x=\sum_{j=1}^{d} k_{j} x_{j}$.

Let $f \in L_{1}\left(\mathbb{T}^{d}\right)$. The Fourier coefficients of $f$ are given by

$$
\widehat{f}(k)=(2 \pi)^{-d} \int_{\mathbb{T}^{d}} f(x) e^{-i k x} d x \quad, \quad k \in \mathbb{Z}^{d} .
$$

We write $F_{d}(w)$ for the Hilbert space of integrable functions on $\mathbb{T}^{d}$ formed by all those $f$ having a finite norm

$$
\left\|f \mid F_{d}(w)\right\|=\left(\sum_{k \in \mathbb{Z}^{d}} w(k)^{2}|\widehat{f}(k)|^{2}\right)^{1 / 2}<\infty .
$$

Here $w(k)>0, k \in \mathbb{Z}^{d}$, are certain weights. If $0<s<\infty, 0<r \leq \infty$ and $w(k)=\left(1+\sum_{j=1}^{d}\left|k_{j}\right|^{r}\right)^{s / r}$, the space $F_{d}(w)$ coincides with the isotropic Sobolev space $H^{s, r}\left(\mathbb{T}^{d}\right)$. The superscript $r$ indicates the norm we are considering in the Sobolev space. When $w(k)=\prod_{j=1}^{d}\left(1+\left|k_{j}\right|^{r}\right)^{s / r}$, we obtain the Sobolev space of dominating mixed smoothness $H_{\text {mix }}^{s, r}\left(\mathbb{T}^{d}\right)$ (see [8] and [20]). 
The Wiener algebra $\mathcal{A}\left(\mathbb{T}^{d}\right)$ consists of all integrable functions on $\mathbb{T}^{d}$ with absolutely convergent Fourier series. The norm on $\mathcal{A}\left(\mathbb{T}^{d}\right)$ is

$$
\left\|f\left|\mathcal{A}\left(\mathbb{T}^{d}\right) \|=\sum_{k \in \mathbb{Z}^{d}}\right| \widehat{f}(k) \mid\right.
$$

The present authors characterized in [8, Theorem 3.1] boundedness and compactness of embeddings of $F_{d}(w)$ in $\mathcal{A}\left(\mathbb{T}^{d}\right), C\left(\mathbb{T}^{d}\right)$ or $L_{\infty}\left(\mathbb{T}^{d}\right)$ :

Theorem 2.1. The following conditions are equivalent.

(i) $F_{d}(w) \hookrightarrow \mathcal{A}\left(\mathbb{T}^{d}\right)$ compactly

(ii) $F_{d}(w) \hookrightarrow \mathcal{A}\left(\mathbb{T}^{d}\right)$ boundedly

(iii) $F_{d}(w) \hookrightarrow C\left(\mathbb{T}^{d}\right)$ compactly

(iv) $F_{d}(w) \hookrightarrow C\left(\mathbb{T}^{d}\right)$ boundedly

(v) $F_{d}(w) \hookrightarrow L_{\infty}\left(\mathbb{T}^{d}\right)$ compactly

(vi) $F_{d}(w) \hookrightarrow L_{\infty}\left(\mathbb{T}^{d}\right)$ boundedly

(vii) $\sum_{k \in \mathbb{Z}^{d}} w(k)^{-2}<\infty$

Owing to this characterization, subsequently we assume that $F_{d}(w)$ is defined by weights satisfying $\sum_{k \in \mathbb{Z}^{d}} w(k)^{-2}<\infty$.

Let $X, Y$ be Banach spaces and $T: X \rightarrow Y$ be a bounded linear operator from $X$ into $Y$. For $n \in \mathbb{N}$, the $n$-th approximation number $a_{n}(T)$ of $T$ is defined by

$$
a_{n}(T)=a_{n}(T: X \rightarrow Y)=\inf \{\|T-R\|: \operatorname{rank} R<n\} .
$$

Here rank $R$ is the dimension of the range of $R$. See [27] and [28] for properties of these numbers.

The following result was proved in [8, Theorem 3.4]. It provides an exact formula for the approximation numbers of the embeddings of Theorem 2.1.

Theorem 2.2. Let $F_{d}(w)$ be given by weights satisfying $\sum_{k \in \mathbb{Z}^{d}} w(k)^{-2}<\infty$, and let $\left(\sigma_{j}\right)_{j \in \mathbb{N}}$ denote the non-increasing rearrangement of $(1 / w(k))_{k \in \mathbb{Z}^{d}}$. Moreover, let

$$
G_{d}=\mathcal{A}\left(\mathbb{T}^{d}\right) \quad \text { or } \quad C\left(\mathbb{T}^{d}\right) \text { or } \quad L_{\infty}\left(\mathbb{T}^{d}\right)
$$

Then one has for all $n \in \mathbb{N}$,

$$
a_{n}\left(I_{d}: F_{d}(w) \rightarrow G_{d}\right)=\left(\sum_{j=n}^{\infty} \sigma_{j}^{2}\right)^{1 / 2}
$$




\section{Embeddings of isotropic Besov spaces}

There is a rich literature on Besov spaces. We refer, for example, to the monographs by Peetre [26] and Triebel [38]. Special emphasis to the periodic situation is given in the monograph by Schmeisser and Triebel [31]. As there, we shall also use the Fourier-analytic approach to introduce these spaces. However, let us mention that they can be described also in terms of moduli of smoothness (differences) or in terms of best approximation by trigonometric polynomials in the sense of equivalent norms. Clearly, for switching to these other characterizations we have to pay a price, expressed by a constant in the related inequalities, which depends in general on $d$. This will not be done here, but it would be of certain interest.

Later on we will be forced to deal with spaces with negative smoothness $s$, that is to say, spaces of tempered distributions. This requires some preparations.

Let $D\left(\mathbb{T}^{d}\right)$ denote the collection of all periodic infinitely differentiable complexvalued functions. In particular, $f(x)=f(y)$ if $x-y=2 \pi k, k \in \mathbb{Z}^{d}$. The locally convex topology in $D\left(\mathbb{T}^{d}\right)$ is generated by the semi-norms

$$
\|f\|_{\alpha}=\sup _{x \in \mathbb{T}^{d}}\left|D^{\alpha} f(x)\right|,
$$

where $\alpha \in \mathbb{N}_{0}^{d}$ with $\mathbb{N}_{0}=\mathbb{N} \cup\{0\}$, and $D^{\alpha} f$ is the distributional derivative of $f$ of order $\alpha$. By $D^{\prime}\left(\mathbb{T}^{d}\right)$ we denote the topological dual of $D\left(\mathbb{T}^{d}\right)$, i.e., the set of all linear functionals $g$ on $D\left(\mathbb{T}^{d}\right)$ such that

$$
|g(f)| \leq c_{N} \sum_{|\alpha| \leq N}\|f\|_{\alpha}
$$

for all $f \in D\left(\mathbb{T}^{d}\right)$ and for some $N \in \mathbb{N}_{0}$ and $c_{N}>0$. We equip $D^{\prime}\left(\mathbb{T}^{d}\right)$ with the weak topology, i.e.,

$$
g=\lim _{j \rightarrow \infty} g_{j} \quad \Longleftrightarrow \quad g(f)=\lim _{j \rightarrow \infty} g_{j}(f) \quad \text { for all } \quad f \in D\left(\mathbb{T}^{d}\right) .
$$

The Fourier coefficients of $g \in D^{\prime}\left(\mathbb{T}^{d}\right)$ are defined by

$$
\widehat{g}(k)=(2 \pi)^{-d} g\left(e^{-i k x}\right), \quad k \in \mathbb{Z}^{d} .
$$

For every $g \in D^{\prime}\left(\mathbb{T}^{d}\right)$ there are a constant $c_{g}>0$ and a natural number $K_{g}$ with

$$
|\widehat{g}(k)| \leq c_{g}(1+|k|)^{K_{g}}, \quad k \in \mathbb{Z}^{d} .
$$

Furthermore, every $g \in D^{\prime}\left(\mathbb{T}^{d}\right)$ can be represented by its Fourier series,

$$
\left.g=\sum_{k \in \mathbb{Z}^{d}} \widehat{g}(k) e^{i k x} \quad \text { (convergence in } D^{\prime}\left(\mathbb{T}^{d}\right)\right) .
$$

For all these facts we refer to [31, 3.2.1 and 3.2.2], but see also [14, Chapter 12].

Starting point of the Fourier-analytic approach to Besov spaces is a smooth dyadic decomposition of unity. By $B_{r}$ we denote the Euclidean ball in $\mathbb{R}^{d}$ of radius $r$, centered at the origin. Let $\psi \in C_{0}^{\infty}\left(\mathbb{R}^{d}\right)$ be a real-valued non-negative function such that

$\operatorname{supp} \psi \subset B_{3 / 2}, \quad \psi(\xi / 2)-\psi(\xi) \geq 0$ for all $\xi \in \mathbb{R}^{d} \quad$ and $\quad \psi(\xi)=1$ for $\xi \in B_{1}$. 
We define $\varphi(\xi)=\psi(\xi / 2)-\psi(\xi)$,

$$
\varphi_{0}(\xi)=\psi(\xi) \quad \text { and } \quad \varphi_{j}(\xi):=\varphi\left(2^{-j+1} \xi\right), \quad j \in \mathbb{N} .
$$

Then

$$
\sum_{j=0}^{\infty} \varphi_{j}(\xi)=1 \quad \text { for all } \quad \xi \in \mathbb{R}^{d}
$$

and

$$
\operatorname{supp} \varphi_{j} \subset B_{3 \cdot 2^{j-1}} \backslash B_{2^{j-1}}, \quad j \in \mathbb{N} .
$$

In addition we mention that in every point $x \in \mathbb{R}^{d}$ at most two of these functions $\varphi_{j}, j \in \mathbb{N}_{0}$, do not vanish. For $g \in D^{\prime}\left(\mathbb{T}^{d}\right)$ we define

$$
g_{j}(x):=\sum_{k \in \mathbb{Z}^{d}} \varphi_{j}(k) \widehat{g}(k) e^{i k x} .
$$

Since $\varphi_{j}$ has compact support, the $g_{j}$ are trigonometric polynomials.

Definition 3.1. Let $1 \leq p, q \leq \infty$ and $s \in \mathbb{R}$. Then the periodic Besov space $B_{p, q}^{s}\left(\mathbb{T}^{d}\right)$ is the collection of all $g \in D^{\prime}\left(\mathbb{T}^{d}\right)$ such that

$$
\left\|g \mid B_{p, q}^{s}\left(\mathbb{T}^{d}\right)\right\|^{\psi}:=\left(\sum_{j=0}^{\infty} 2^{j s q}\left\|\sum_{k \in \mathbb{Z}^{d}} \varphi_{j}(k) \widehat{g}(k) e^{i k x} \mid L_{p}\left(\mathbb{T}^{d}\right)\right\|^{q}\right)^{1 / q}<\infty .
$$

Remark 3.2. (i) Of course, the norm in (3.5) depends on the generating function $\psi$ of the chosen smooth decomposition of unity $\left(\varphi_{j}\right)_{j}$. All these norms are equivalent. If necessary, we indicate the dependence on $\psi$ by a superscript as in (3.5). Otherwise we simply write $\left\|g \mid B_{p, q}^{s}\left(\mathbb{T}^{d}\right)\right\|$.

(ii) The spaces $B_{p, q}^{s}\left(\mathbb{T}^{d}\right)$ are Banach spaces such that

$$
D\left(\mathbb{T}^{d}\right) \hookrightarrow B_{p, q}^{s}\left(\mathbb{T}^{d}\right) \hookrightarrow D^{\prime}\left(\mathbb{T}^{d}\right),
$$

see [31, Theorem 3.5.1].

(iii) Let us also mention that we have, in the sense of equivalent norms,

$$
H^{s, r}\left(\mathbb{T}^{d}\right)=B_{2,2}^{s}\left(\mathbb{T}^{d}\right),
$$

where the equivalence constants depend on $\psi, s, d$ and $r$.

By convention if the equivalence class of a measurable function $f$ contains a continuous representative, then we call $f$ itself continuous and work with the continuous representative.

Lemma 3.3. We have the following chain of continuous embeddings

$$
B_{2,1}^{d / 2}\left(\mathbb{T}^{d}\right) \hookrightarrow \mathcal{A}\left(\mathbb{T}^{d}\right) \hookrightarrow B_{\infty, 1}^{0}\left(\mathbb{T}^{d}\right) \hookrightarrow C\left(\mathbb{T}^{d}\right),
$$

where the last two embedding operators have norm one. 
Proof. The embedding $B_{2,1}^{d / 2}\left(\mathbb{T}^{d}\right) \hookrightarrow \mathcal{A}\left(\mathbb{T}^{d}\right)$ is well-known. We refer for example to the book of Triebel [37, page 141 in Section 3.4.3]. Concerning the second embedding, by (3.3) we have for $g \in \mathcal{A}\left(\mathbb{T}^{d}\right)$ the inequality

$$
\sum_{j=0}^{\infty}\left\|\sum_{k \in \mathbb{Z}^{d}} \varphi_{j}(k) \widehat{g}(k) e^{i k x}\left|L_{\infty}\left(\mathbb{T}^{d}\right)\left\|\leq \sum_{j=0}^{\infty} \sum_{k \in \mathbb{Z}^{d}} \varphi_{j}(k)|\widehat{g}(k)| \leq\right\| g\right| \mathcal{A}\left(\mathbb{T}^{d}\right)\right\| .
$$

Here we used the non-standard condition $\varphi_{j} \geq 0$, see (3.1).

Finally, we consider the embedding $B_{\infty, 1}^{0}\left(\mathbb{T}^{d}\right) \hookrightarrow C\left(\mathbb{T}^{d}\right)$. For any $g \in$ $B_{\infty, 1}^{0}\left(\mathbb{T}^{d}\right)$, the continuous representative in the equivalence class of $g$ is just the Fourier series of $g$. It follows

$$
\begin{aligned}
|g(x)| & =\left|\sum_{k \in \mathbb{Z}^{d}}\left(\sum_{j=0}^{\infty} \varphi_{j}(k)\right) \widehat{g}(k) e^{i k x}\right| \\
& \leq \sum_{j=0}^{\infty}\left\|\sum_{k \in \mathbb{Z}^{d}} \varphi_{j}(k) \widehat{g}(k) e^{i k x}\left|L_{\infty}\left(\mathbb{T}^{d}\right)\|\leq\| g\right| B_{\infty, 1}^{0}\left(\mathbb{T}^{d}\right)\right\| .
\end{aligned}
$$

In both cases this proves that the norm of the corresponding embedding operator is $\leq 1$. By considering the constant function $g(x)=1$, it is immediate that these norms are $\geq 1$ as well.

Remark 3.4. (i) The embeddings stated in (3.6) cannot be improved in the framework of periodic Besov spaces. Indeed, if we replace $B_{2,1}^{d / 2}\left(\mathbb{T}^{d}\right)$ by $B_{2, q}^{d / 2}\left(\mathbb{T}^{d}\right)$ for some $q>1$, then an embedding into $\mathcal{A}\left(\mathbb{T}^{d}\right)$ cannot exist, since $B_{2, q}^{d / 2}\left(\mathbb{T}^{d}\right)$ contains unbounded functions, see [37, 3.4.3] and [33]. Also $B_{\infty, 1}^{0}\left(\mathbb{T}^{d}\right)$ cannot be replaced by $B_{\infty, q}^{0}\left(\mathbb{T}^{d}\right)$ with $q<1$ in the embedding $\mathcal{A}\left(\mathbb{T}^{d}\right) \hookrightarrow B_{\infty, 1}^{0}\left(\mathbb{T}^{d}\right)$. There are explicit counterexamples, we omit the details.

(ii) We did not indicate the chosen norm in (3.6) in the Besov spaces. The statement is true with respect to all norms of the type $\left\|\cdot \mid B_{\infty, 1}^{0}\left(\mathbb{T}^{d}\right)\right\|^{\psi}$, see (3.5).

As an immediate consequence of Lemma 3.3 and Theorem 2.2 we obtain

Theorem 3.5. Let $F_{d}(w)$ be given by a weight $w$ satisfying $\sum_{k \in \mathbb{Z}^{d}} w(k)^{-2}<\infty$ and let $\left(\sigma_{j}\right)_{j \in \mathbb{N}}$ be the non-increasing rearrangement of $(1 / w(k))_{k \in \mathbb{Z}^{d}}$. Then one has for all $n \in \mathbb{N}$

$$
\begin{aligned}
a_{n}\left(I_{d}: F_{d}(w) \rightarrow B_{\infty, 1}^{0}\left(\mathbb{T}^{d}\right)\right) & =a_{n}\left(I_{d}: F_{d}(w) \rightarrow L_{\infty}\left(\mathbb{T}^{d}\right)\right) \\
& =a_{n}\left(I_{d}: F_{d}(w) \rightarrow C\left(\mathbb{T}^{d}\right)\right) \\
& =a_{n}\left(I_{d}: F_{d}(w) \rightarrow \mathcal{A}\left(\mathbb{T}^{d}\right)\right)=\left(\sum_{j=n}^{\infty} \sigma_{j}^{2}\right)^{1 / 2} .
\end{aligned}
$$

This result is true for any norm of the type $\left\|\cdot \mid B_{\infty, 1}^{0}\left(\mathbb{T}^{d}\right)\right\|^{\psi}$ on $B_{\infty, 1}^{0}\left(\mathbb{T}^{d}\right)$. See Definition 3.1. 


\section{Embeddings of Besov spaces of dominating mixed smoothness}

As mentioned in the Introduction, Sobolev and Besov spaces of dominating mixed smoothness are helpful when dealing with approximation problems in high dimensions. These spaces are much smaller than their isotropic counterparts. The behaviour of the approximation numbers of embeddings of isotropic spaces into $L_{p}\left(\mathbb{T}^{d}\right)$ is closer to the one-dimensional case than to the $d$-dimensional situation.

Let $\psi \in C_{0}^{\infty}(\mathbb{R})$ be a real-valued non-negative function satisfying properties (3.1) in the case $d=1$. The associated smooth dyadic decomposition of unity on $\mathbb{R}$ is denoted by $\left(\varphi_{j}\right)_{j=0}^{\infty}$, see $(3.2)$. We shall work with tensor products of this decomposition: For $\ell=\left(\ell_{1}, \ldots, \ell_{d}\right) \in \mathbb{N}_{0}^{d}$ and $x=\left(x_{1}, \ldots, x_{d}\right) \in \mathbb{R}^{d}$, we put

$$
\varphi_{\ell}(x)=\prod_{j=1}^{d} \varphi_{\ell_{j}}\left(x_{j}\right)
$$

Observe that

$$
\sum_{\ell \in \mathbb{N}_{0}^{d}} \varphi_{\ell}(x)=1 \quad \text { for all } \quad x \in \mathbb{R}^{d},
$$

and that now for every $x \in \mathbb{R}^{d}$ at most $2^{d}$ functions $\varphi_{\ell}$ do not vanish.

Definition 4.1. Let $1 \leq p, q \leq \infty$ and $t \in \mathbb{R}$. Then the periodic Besov space $S_{p, q}^{t} B\left(\mathbb{T}^{d}\right)$ of dominating mixed smoothness is the collection of all $g \in D^{\prime}\left(\mathbb{T}^{d}\right)$ such that the norm

$$
\left\|g \mid S_{p, q}^{t} B\left(\mathbb{T}^{d}\right)\right\|:=\left(\sum_{\ell \in \mathbb{N}_{0}^{d}} 2^{|\ell|_{1} t q}\left\|\sum_{k \in \mathbb{Z}^{d}} \varphi_{\ell}(k) \widehat{g}(k) e^{i k x} \mid L_{p}\left(\mathbb{T}^{d}\right)\right\|^{q}\right)^{1 / q}
$$

is finite.

Remark 4.2. (i) Of course, the norm in (4.1) depends on the generating function $\psi$ of the chosen smooth decomposition of unity $\left(\varphi_{j}\right)_{j}$. Again all these norms are equivalent. If necessary, we indicate the dependence on $\psi$ as in (3.5) by $\left\|g \mid S_{p, q}^{t} B\left(\mathbb{T}^{d}\right)\right\|^{\psi}$.

(ii) The spaces $S_{p, q}^{t} B\left(\mathbb{T}^{d}\right)$ are Banach spaces such that

$$
D\left(\mathbb{T}^{d}\right) \hookrightarrow S_{p, q}^{t} B\left(\mathbb{T}^{d}\right) \hookrightarrow D^{\prime}\left(\mathbb{T}^{d}\right)
$$

(iii) Let us also mention that, in the sense of equivalent norms,

$$
H_{\text {mix }}^{s, r}\left(\mathbb{T}^{d}\right)=S_{2,2}^{s} B\left(\mathbb{T}^{d}\right),
$$

where the equivalence constants depend on $\psi, s, r$ and $d$.

(iv) One of the most attractive features of these spaces is the following. If

$$
g(x)=\prod_{j=1}^{d} g_{j}\left(x_{j}\right), \quad x=\left(x_{1}, \ldots, x_{d}\right) \in \mathbb{T}^{d},
$$

then

$$
\left\|g\left|S_{p, q}^{t} B\left(\mathbb{T}^{d}\right)\left\|^{\psi}=\prod_{j=1}^{d}\right\| g_{j}\right| B_{p, q}^{t}(\mathbb{T})\right\|^{\psi}
$$


(v) Besov spaces of dominating mixed smoothness are investigated since the seventies of the last century, we refer to the monographs of Amanov [3] and of Schmeisser, Triebel [31] as well as to the article of Lizorkin and Nikol'skij [22].

Lemma 4.3. We have the chain of continuous embeddings

$$
\mathcal{A}\left(\mathbb{T}^{d}\right) \hookrightarrow S_{\infty, 1}^{0} B\left(\mathbb{T}^{d}\right) \hookrightarrow C\left(\mathbb{T}^{d}\right)
$$

where the embedding operators are of norm one.

Proof. For $g \in \mathcal{A}\left(\mathbb{T}^{d}\right)$, using (3.3), we have

$$
\begin{aligned}
\sum_{\ell \in \mathbb{N}_{0}^{d}} \| & \sum_{k \in \mathbb{Z}^{d}} \varphi_{\ell}(k) \widehat{g}(k) e^{i k x} \mid L_{\infty}\left(\mathbb{T}^{d}\right) \| \\
& \leq \sum_{k \in \mathbb{Z}^{d}}\left[\sum_{\ell \in \mathbb{N}_{0}^{d}} \varphi_{\ell}(k)\right]|\widehat{g}(k)| \leq\left\|g \mid \mathcal{A}\left(\mathbb{T}^{d}\right)\right\| .
\end{aligned}
$$

Next, we turn to the embedding $S_{\infty, 1}^{0} B\left(\mathbb{T}^{d}\right) \hookrightarrow C\left(\mathbb{T}^{d}\right)$. It follows

$$
\begin{aligned}
|g(x)| & =\left|\sum_{k \in \mathbb{Z}^{d}}\left[\sum_{\ell \in \mathbb{N}_{0}^{d}} \varphi_{\ell}(k)\right] \widehat{g}(k) e^{i k x}\right| \\
& \leq \sum_{\ell \in \mathbb{N}_{0}^{d}}\left\|\sum_{k \in \mathbb{Z}^{d}} \varphi_{\ell}(k) \widehat{g}(k) e^{i k x}\left|L_{\infty}\left(\mathbb{T}^{d}\right)\|=\| g\right| S_{\infty, 1}^{0} B\left(\mathbb{T}^{d}\right)\right\| .
\end{aligned}
$$

In both cases this proves that the norm of the associated embedding operator is $\leq 1$. Considering $g(x)=1$, it is immediate that these norms are $\geq 1$ as well.

Similarly as for isotropic Besov spaces (see Theorem 3.5), we can derive the following consequence from Theorem 2.2, where we can use any norm of the type $\left\|g \mid S_{p, q}^{0} B\left(\mathbb{T}^{d}\right)\right\|^{\psi}$.

Theorem 4.4. Let $F_{d}(w)$ be given by a weight $w$ satisfying $\sum_{k \in \mathbb{Z}^{d}} w(k)^{-2}<\infty$, and let $\left(\sigma_{j}\right)_{j \in \mathbb{N}}$ be the non-increasing rearrangement of $(1 / w(k))_{k \in \mathbb{Z}^{d}}$. Then one has for all $n \in \mathbb{N}$

$$
\begin{aligned}
a_{n}\left(I_{d}: F_{d}(w) \rightarrow S_{\infty, 1}^{0} B\left(\mathbb{T}^{d}\right)\right) & =a_{n}\left(I_{d}: F_{d}(w) \rightarrow L_{\infty}\left(\mathbb{T}^{d}\right)\right) \\
& =a_{n}\left(I_{d}: F_{d}(w) \rightarrow C\left(\mathbb{T}^{d}\right)\right) \\
& =a_{n}\left(I_{d}: F_{d}(w) \rightarrow \mathcal{A}\left(\mathbb{T}^{d}\right)\right)=\left(\sum_{j=n}^{\infty} \sigma_{j}^{2}\right)^{1 / 2} .
\end{aligned}
$$

Next we deal with another situation where now one has some advantage from the flexibility in the target space. Duality will be an important tool for our arguments. Therefore we define

$$
\mathcal{B}\left(\mathbb{T}^{d}\right)=\left\{g \in D^{\prime}\left(\mathbb{T}^{d}\right):\left\|g\left|\mathcal{B}\left(\mathbb{T}^{d}\right) \|=\sup _{k \in \mathbb{Z}^{d}}\right| \widehat{g}(k) \mid<\infty\right\}\right.
$$

Obviously we have, with equality of norms, the duality

$$
\left(\mathcal{A}\left(\mathbb{T}^{d}\right)\right)^{\prime}=\mathcal{B}\left(\mathbb{T}^{d}\right)
$$


Let $w=(w(k))_{k \in \mathbb{Z}^{d}}$ be a positive weight. If $F_{d}(w)$ is defined as in (2.1) then

$$
\left(F_{d}(w)\right)^{\prime}=F_{d}(1 / w)
$$

with equality of norms. All these dual spaces are subspaces of $D^{\prime}\left(\mathbb{T}^{d}\right)$ (since $D\left(\mathbb{T}^{d}\right)$ is dense in the original spaces).

Next we recall a result due to Hutton [15] (see also [27, Theorem 11.7.4] and [7, Proposition 2.5.2]). If $T: X \rightarrow Y$ is a compact linear operator between arbitrary Banach spaces, then

$$
a_{n}(T)=a_{n}\left(T^{\prime}\right)
$$

where $T^{\prime}: Y^{\prime} \rightarrow X^{\prime}$ denotes the dual operator of $T$. Besides this symmetry property, in our later computations it will be also useful the so-called ideal property: If the operators $T: X \rightarrow Y, R: Y \rightarrow Z$ and $S: Z \rightarrow W$ are bounded, then $a_{n}(S R T) \leq\|T: X \rightarrow Y\| a_{n}(R)\|S: Z \rightarrow W\|$.

The next result follows by combining the property of symmetry with Theorem 2.2

Corollary 4.5. Let $F_{d}(w)$ be given by a weight $w$ satisfying $\sum_{k \in \mathbb{Z}^{d}} w(k)^{-2}<\infty$.

Then one has for all $n \in \mathbb{N}$

$$
\begin{aligned}
a_{n}\left(I_{d}: \mathcal{B}\left(\mathbb{T}^{d}\right) \rightarrow F_{d}(1 / w)\right) & =a_{n}\left(I_{d}: F_{d}(w) \rightarrow \mathcal{A}\left(\mathbb{T}^{d}\right)\right) \\
& =a_{n}\left(I_{d}: F_{d}(w) \rightarrow L_{\infty}\left(\mathbb{T}^{d}\right)\right) \\
& =a_{n}\left(I_{d}: L_{1}\left(\mathbb{T}^{d}\right) \rightarrow F_{d}(1 / w)\right) .
\end{aligned}
$$

Proof. The second equality is due to Theorem 2.2, and the other two equalities follow by duality.

The following relation holds between $S_{1, \infty}^{0} B\left(\mathbb{T}^{d}\right)$ and $\mathcal{B}\left(\mathbb{T}^{d}\right)$.

Lemma 4.6. (i) For all $g \in S_{1, \infty}^{0} B\left(\mathbb{T}^{d}\right)$ we have

$$
|\widehat{g}(k)| \leq 2^{d}\left\|g \mid S_{1, \infty}^{0} B\left(\mathbb{T}^{d}\right)\right\|, \quad k \in \mathbb{Z}^{d} .
$$

(ii) It holds $\left\|I_{d}: S_{1, \infty}^{0} B\left(\mathbb{T}^{d}\right) \longrightarrow \mathcal{B}\left(\mathbb{T}^{d}\right)\right\|=2^{d}$ independent of the decomposition of unity as defined at the beginning of this section.

Proof. Step 1. Proof of (i). For every $k \in \mathbb{Z}^{d}$ we consider the index set

$$
L_{k}=\left\{\ell \in \mathbb{N}_{0}^{d}: \varphi_{\ell}(k)>0\right\} .
$$

By assumption (3.1) on $\psi$, we have $\operatorname{card}\left(L_{k}\right) \leq 2^{d}$ (see the comment before Definition 4.1).

For $\ell \in \mathbb{N}_{0}^{d}$, consider the trigonometric polynomials

$$
g_{\ell}(x)=\sum_{k \in \mathbb{Z}^{d}} \varphi_{\ell}(k) \widehat{g}(k) e^{i k x}
$$

Then $\widehat{g}_{\ell}(k)=\varphi_{\ell}(k) \widehat{g}(k)$ and

$$
\begin{aligned}
|\widehat{g}(k)| & =\left|\sum_{\ell \in \mathbb{N}_{0}^{d}} \varphi_{\ell}(k) \widehat{g}(k)\right| \leq \sum_{\ell \in L_{k}}\left|\widehat{g_{\ell}}(k)\right| \\
& \leq \sum_{\ell \in L_{k}}\left\|g_{\ell}\left|L_{1}\left(\mathbb{T}^{d}\right)\left\|\leq 2^{d}\right\| g\right| S_{1, \infty}^{0} B\left(\mathbb{T}^{d}\right)\right\| .
\end{aligned}
$$


Step 2. Proof of (ii). From Step 1 it follows $\left\|I_{d}: S_{1, \infty}^{0} B\left(\mathbb{T}^{d}\right) \longrightarrow \mathcal{B}\left(\mathbb{T}^{d}\right)\right\| \leq 2^{d}$. To show equality we proceed as follows. Let $\psi \in C_{0}^{\infty}(\mathbb{R})$ be the generator of our univariate smooth dyadic decomposition of unity in (3.1). For a moment $\left(\varphi_{\ell}\right)_{\ell=0}^{\infty}$ denotes the associated univariate decomposition, see (3.2). By assumption there exists a point $t_{1} \in(2,4)$ such that $\varphi_{1}\left(t_{1}\right)=\varphi_{2}\left(t_{1}\right)=1 / 2$. Now we shift this point by defining $t_{j}:=2^{j-1} t_{1}, j \in \mathbb{N}$. Because of $(3.2)$ it follows $t_{j} \in\left(2^{j}, 2^{j+1}\right)$ and $\varphi_{j}\left(t_{j}\right)=\varphi_{j+1}\left(t_{j}\right)=1 / 2$. Clearly to each $j \geq 1$ there exists a natural number $k_{j}$ such that $\left|t_{j}-k_{j}\right| \leq 1$. We have

$$
\left|\varphi_{j}\left(t_{j}\right)-\varphi_{j}\left(k_{j}\right)\right|=\left|\varphi_{1}\left(t_{1}\right)-\varphi_{1}\left(2^{-j+1} k_{j}\right)\right| \leq\left\|\varphi_{1}^{\prime} \mid L_{\infty}(\mathbb{R})\right\| \cdot 2^{-j+1} .
$$

Hence, for any $\varepsilon>0$ there exists a natural number $j_{0}$ with

$$
\left|\varphi_{j}\left(t_{j}\right)-\varphi_{j}\left(k_{j}\right)\right|+\left|\varphi_{j+1}\left(t_{j}\right)-\varphi_{j+1}\left(k_{j}\right)\right|<\varepsilon, \quad j \geq j_{0} .
$$

Now we turn to the tensor product system $\left(\varphi_{\ell}\right)_{\ell \in \mathbb{N}_{0}^{d}}$. When writing $\bar{m}, m \in \mathbb{N}$, we mean the vector $(m, \ldots, m) \in \mathbb{N}_{0}^{d}$. We define

$$
x^{m}:=\left(t_{m}, \ldots, t_{m}\right) \quad \text { and } \quad k^{m}:=\left(k_{m}, \ldots, k_{m}\right), \quad m \in \mathbb{N} .
$$

It follows

$$
\left|\varphi_{\bar{m}}\left(x^{m}\right)-\varphi_{\bar{m}}\left(k^{m}\right)\right|+\left|\varphi_{\overline{m+1}}\left(x^{m}\right)-\varphi_{\overline{m+1}}\left(k^{m}\right)\right|<\varepsilon, \quad m \geq m_{0},
$$

if $m_{0}=m_{0}(\varepsilon)$ is chosen large enough. Next, as test functions, we choose $f_{m}(x):=e^{i k^{m} x}, m \in \mathbb{N}$. Then

$$
\left\|f_{m}\left|\mathcal{B}\left(\mathbb{T}^{d}\right)\right|\right\|=1
$$

and

$$
\left.\| f_{m} \mid S_{1, \infty}^{0} B\left(\mathbb{T}^{d}\right)\right) \mid \| \geq 2^{-d}-\varepsilon, \quad m \geq m_{1},
$$

if $m_{1}=m_{1}(\varepsilon)$ is chosen large enough. Hence we have proved $\left\|I_{d}: S_{1, \infty}^{0} B\left(\mathbb{T}^{d}\right) \longrightarrow \mathcal{B}\left(\mathbb{T}^{d}\right)\right\| \geq 2^{d}$.

As a direct consequence of Lemma 4.6 and the ideal property of approximation numbers, we get the following.

Lemma 4.7. Let $F_{d}(w)$ be given by a weight $w$ satisfying $\sum_{k \in \mathbb{Z}^{d}} w(k)^{-2}<\infty$. Then one has for all $n \in \mathbb{N}$

$$
a_{n}\left(I_{d}: S_{1, \infty}^{0} B\left(\mathbb{T}^{d}\right) \rightarrow F_{d}(1 / w)\right) \leq 2^{d} a_{n}\left(I_{d}: \mathcal{B}\left(\mathbb{T}^{d}\right) \rightarrow F_{d}(1 / w)\right) .
$$

In what follows, we work mainly with $w(k)=\prod_{j=1}^{d}\left(1+\left|k_{j}\right|^{2}\right)^{1 / 2}$. Let $\sigma \in \mathbb{R}$. For the dominating mixed case that we are dealing with, the standard lifting operator is given by

$$
J_{\text {mix }}^{\sigma}: g \rightarrow \sum_{k \in \mathbb{Z}^{d}} \widehat{g}(k) w(k)^{-\sigma} e^{i k x}=\sum_{k \in \mathbb{Z}^{d}} \widehat{g}(k) \prod_{j=1}^{d}\left(1+\left|k_{j}\right|^{2}\right)^{-\sigma / 2} e^{i k x} .
$$

It is obvious that $J_{\text {mix }}^{\sigma}$ is an isometry from $H_{\text {mix }}^{s, 2}\left(\mathbb{T}^{d}\right)$ onto $H_{\text {mix }}^{s+\sigma, 2}\left(\mathbb{T}^{d}\right)$.

Lemma 4.8. Let $\sigma \in \mathbb{R}, 1 \leq p, q \leq \infty$ and $t \in \mathbb{R}$. Then $J_{\text {mix }}^{\sigma}$ maps $S_{p, q}^{t} B\left(\mathbb{T}^{d}\right)$ isomorphically onto $S_{p, q}^{t+\sigma} B\left(\mathbb{T}^{d}\right)$. 
Proof. One can follow the arguments used in the proof of [38, Theorem 2.3.8] which describes the isotropic situation.

Next we establish two norm estimates involving the lifting operator. The first one extends Lemma 4.6 to spaces $S_{1, \infty}^{t} B\left(\mathbb{T}^{d}\right)$ with $t>0$.

Theorem 4.9. Let $t>0$. Then

$$
\left\|J_{\text {mix }}^{-t}: S_{1, \infty}^{t} B\left(\mathbb{T}^{d}\right) \longrightarrow \mathcal{B}\left(\mathbb{T}^{d}\right)\right\| \leq 2^{d}(3 / 2)^{t d}
$$

Proof. Let $g_{\ell}$ by the function defined in (4.4). Since $J_{\text {mix }}^{-t} g(x)=\sum_{k \in \mathbb{Z}^{d}} \widehat{g}(k) w(k)^{t} e^{i k x}$, for any $k \in \mathbb{Z}^{d}$, we obtain

$$
\begin{aligned}
\left|\widehat{J_{\text {mix }}^{-t} g}(k)\right| & =w(k)^{t}|\widehat{g}(k)|=w(k)^{t}\left|\sum_{\ell \in \mathbb{N}_{0}^{d}} \varphi_{\ell}(k) \widehat{g}(k)\right| \\
& =w(k)^{t}\left|\sum_{\ell \in \mathbb{N}_{0}^{d}} \widehat{g_{\ell}}(k)\right| \\
& =w(k)^{t}\left|\sum_{\ell \in L_{k}} \widehat{g_{\ell}}(k)\right|
\end{aligned}
$$

where $L_{k}$ is the set defined in (4.3). Using that $\left|\widehat{g}_{\ell}(k)\right| \leq\left\|g_{\ell} \mid L_{1}\left(\mathbb{T}^{d}\right)\right\|$, we obtain

$$
\begin{aligned}
\left|\widehat{J_{\text {mix }}^{-t} g}(k)\right| & \leq w(k)^{t} \sum_{\ell \in L_{k}} 2^{-|\ell|_{1} t_{2}|\ell|_{1} t}\left\|g_{\ell} \mid L_{1}\right\| \\
& \leq\left(w(k)^{t} \sum_{\ell \in L_{k}} 2^{-|\ell|_{1} t}\right)\left\|g \mid S_{1, \infty}^{t} B\left(\mathbb{T}^{d}\right)\right\| .
\end{aligned}
$$

It remains to compute the factor in brackets.

For $1 \leq j \leq d$, let $L_{k_{j}}=\left\{\ell_{j} \in \mathbb{N}_{0}: \varphi_{\ell_{j}}\left(k_{j}\right)>0\right\}$. According to the construction of $\varphi_{\ell_{j}}$, there are at most two $\ell_{j}$ in $L_{k_{j}}$. Moreover, if $\ell_{j} \in \mathbb{N}$ belongs to $L_{k_{j}}$ then $\left|k_{j}\right|<3 \cdot 2^{\ell_{j}-1}$. In this inequality, the number in the right side is integer. Hence $1+\left|k_{j}\right|^{2} \leq\left(3 \cdot 2^{\ell_{j}-1}\right)^{2}$ and so $\left(1+\left|k_{j}\right|^{2}\right)^{t / 2} \leq(3 / 2)^{t} 2^{\ell_{j} t}$. If $\ell_{j}=0$ and $0 \in L_{k_{j}}$ then $\left|k_{j}\right| \leq 1$, which yields that $\left(1+\left|k_{j}\right|^{2}\right)^{t / 2} \leq 2^{t / 2} \leq(3 / 2)^{t}$. Consequently,

$$
w(k)^{t} \sum_{\ell \in L_{k}} 2^{-|\ell|_{1} t}=\prod_{j=1}^{d} \sum_{\ell_{j} \in L_{k_{j}}}\left(1+\left|k_{j}\right|^{2}\right)^{t / 2} 2^{-\ell_{j} t} \leq 2^{d}(3 / 2)^{t d} .
$$

This completes the proof.

In the next result we write $\mathcal{F}$ for the Fourier transform on $\mathbb{R}^{d}$ and $\mathcal{F}^{-1}$ for the inverse Fourier transform. We normalize it as follows

$$
\mathcal{F} f(\xi)=\int_{\mathbb{R}^{d}} f(x) e^{-i x \xi} d x, \quad \xi \in \mathbb{R}^{d} .
$$


Theorem 4.10. Let $t>0$. Then we have

$$
\left\|J_{\text {mix }}^{t}: L_{1}\left(\mathbb{T}^{d}\right) \longrightarrow S_{1, \infty}^{t} B\left(\mathbb{T}^{d}\right)\right\| \leq c(\varphi, t)^{d} 2^{(t+1) d} .
$$

Here

$$
c(\varphi, t)=\max _{j=0,1}\left(\left\|\varphi_{j}^{\prime \prime}\left|L_{1}(\mathbb{R})\|+2 t\| \varphi_{j}^{\prime}\right| L_{1}(\mathbb{R})\right\|+(t(t+3)+1)\left\|\varphi_{j} \mid L_{1}(\mathbb{R})\right\|\right) .
$$

Proof. To make the arguments more transparent, we indicate now the dimension in the weights by writing $w(x)=\left(1+x^{2}\right)^{1 / 2}$ for $x \in \mathbb{R}$ and $w_{d}(x)=$ $w\left(x_{1}\right) \cdots w\left(x_{d}\right)$ for $x \in \mathbb{R}^{d}$.

Let $g \in L_{1}\left(\mathbb{T}^{d}\right)$. We have

$$
\left\|J_{\text {mix }}^{t} g \mid S_{1, \infty}^{t} B\left(\mathbb{T}^{d}\right)\right\|=\sup _{\ell \in \mathbb{N}_{0}^{d}}\left(2^{|\ell|_{1} t}\left\|\sum_{k \in \mathbb{Z}^{d}} \varphi_{\ell}(k) w_{d}(k)^{-t} \widehat{g}(k) e^{i k x} \mid L_{1}\left(\mathbb{T}^{d}\right)\right\|\right) .
$$

Take any $\ell \in \mathbb{N}_{0}^{d}$, we have

$$
\begin{aligned}
\sum_{k \in \mathbb{Z}^{d}} \varphi_{\ell}(k) \frac{\widehat{g}(k)}{w_{d}(k)^{t}} e^{i k x} & =(2 \pi)^{-d} \int_{\mathbb{T}^{d}} g(y) \sum_{k \in \mathbb{Z}^{d}} \frac{\varphi_{\ell}(k)}{w_{d}(k)^{t}} e^{i k(x-y)} d y \\
& =\int_{\mathbb{T}^{d}} g(y) \sum_{m \in \mathbb{Z}^{d}} \mathcal{F}^{-1}\left(\frac{\varphi_{\ell}}{w_{d}^{t}}\right)(x-y+2 \pi m) d y,
\end{aligned}
$$

where the second equality follows by using Poisson's summation formula. Whence,

$$
\begin{aligned}
\| \sum_{k \in \mathbb{Z}^{d}} \varphi_{\ell}(k) & w_{d}(k)^{-t} \widehat{g}(k) e^{i k x} \mid L_{1}\left(\mathbb{T}^{d}\right) \| \\
\leq & (2 \pi)^{-d} \int_{\mathbb{T}^{d}} \sum_{m \in \mathbb{Z}^{d}} \int_{\mathbb{T}^{d}}\left|\mathcal{F}^{-1}\left(\frac{\varphi_{\ell}}{w_{d}^{t}}\right)(x-y+2 \pi m)\right| d x|g(y)| d y \\
& =(2 \pi)^{-d} \int_{\mathbb{T}^{d}} \int_{\mathbb{R}^{d}}\left|\mathcal{F}^{-1}\left(\frac{\varphi_{\ell}}{w_{d}^{t}}\right)(z)\right| d z|g(y)| d y \\
& =\left\|\mathcal{F}^{-1}\left(\frac{\varphi_{\ell}}{w_{d}^{t}}\right)\left|L_{1}\left(\mathbb{R}^{d}\right)\|\| g\right| L_{1}\left(\mathbb{T}^{d}\right)\right\| \\
& =\prod_{j=1}^{d}\left\|\mathcal{F}^{-1}\left(\frac{\varphi_{\ell_{j}}}{w^{t}}\right)\left|L_{1}(\mathbb{R})\|\| g\right| L_{1}\left(\mathbb{T}^{d}\right)\right\| .
\end{aligned}
$$

Therefore, it suffices to show that for any $\ell \in \mathbb{N}_{0}$ we have

$$
2^{\ell t}\left\|\mathcal{F}^{-1}\left(\frac{\varphi_{\ell}}{w^{t}}\right) \mid L_{1}(\mathbb{R})\right\| \leq c(\varphi, t) 2^{t+1} .
$$

Assume first that $\ell \in \mathbb{N}$. We have

$$
2^{\ell t}\left\|\mathcal{F}^{-1}\left(\frac{\varphi_{\ell}}{w^{t}}\right)\left|L_{1}(\mathbb{R}) \|=2^{t} \int_{-\infty}^{\infty}\right| \int_{-\infty}^{\infty} \frac{\varphi_{\ell}(x) 2^{(\ell-1) t}}{w(x)^{t}} e^{i x y} d x \mid d y .\right.
$$

Using the substitution $u=2^{-\ell+1}$ in the inner integral and $z=2^{\ell-1} y$ in the 
outer one, we get

$$
\begin{gathered}
\int_{-\infty}^{\infty}\left|\int_{-\infty}^{\infty} \frac{\varphi_{\ell}(x) 2^{(\ell-1) t}}{w(x)^{t}} e^{i x y} d x\right| d y=\int_{-\infty}^{\infty}\left|\int_{-\infty}^{\infty} \frac{\varphi_{1}(u) 2^{(\ell-1) t}}{w\left(2^{\ell-1} u\right)^{t}} e^{i u z} d u\right| d z \\
=\int_{-\infty}^{\infty}\left|\int_{-\infty}^{\infty} \frac{\varphi_{1}(u)}{\left(u^{2}+2^{-2(\ell-1)}\right)^{t / 2}} e^{i u z} d u\right| d z \\
=\int_{|z| \leq 1}\left|\int_{-\infty}^{\infty} \frac{\varphi_{1}(u)}{\left(u^{2}+2^{-2(\ell-1)}\right)^{t / 2}} e^{i u z} d u\right| d z \\
\quad+\int_{|z| \geq 1}\left|\int_{-\infty}^{\infty} \frac{\varphi_{1}(u)}{\left(u^{2}+2^{-2(\ell-1)}\right)^{t / 2}} e^{i u z} d u\right| d z \\
=I_{1}+I_{2} .
\end{gathered}
$$

The integral $I_{1}$ can be estimated easily by observing that $\operatorname{supp} \varphi_{1} \subseteq\{u \in \mathbb{R}$ : $1 \leq|u| \leq 3\}$. We get

$$
\begin{aligned}
I_{1} & \leq \int_{|z| \leq 1} \int_{1 \leq|u| \leq 3}\left|\frac{\varphi_{1}(u)}{\left(u^{2}+2^{-2(\ell-1)}\right)^{t / 2}} e^{i u z}\right| d u d z \\
& \leq \int_{|z| \leq 1} \int_{1 \leq|u| \leq 3} \frac{\varphi_{1}(u)}{|u|^{t}} d u d z \leq 2\left\|\varphi_{1} \mid L_{1}(\mathbb{R})\right\| .
\end{aligned}
$$

Now we proceed with $I_{2}$. The function $h(u)=\varphi(u)\left(u^{2}+2^{-2(\ell-1)}\right)^{-t / 2}$ has compact support. Hence, using twice integration by parts in the inner integral, we obtain

$$
\begin{aligned}
\int_{-\infty}^{\infty} \frac{\varphi_{1}(u)}{\left(u^{2}+2^{-2(\ell-1)}\right)^{t / 2}} e^{i u z} d u & =\int_{-\infty}^{\infty} h(u) e^{i u z} d u \\
& =-\int_{-\infty}^{\infty} h^{\prime}(u) \frac{e^{i u z}}{i z} d u=\int_{-\infty}^{\infty} h^{\prime \prime}(u) \frac{e^{i u z}}{(i z)^{2}} d u
\end{aligned}
$$

whence

$$
\begin{aligned}
I_{2} & =\int_{|z| \geq 1}\left|\int_{-\infty}^{\infty} h(u) e^{i u z} d u\right| d z=\int_{|z| \geq 1}\left|\int_{-\infty}^{\infty} h^{\prime \prime}(u) e^{i u z} d u\right| \frac{d z}{z^{2}} \\
& \leq \int_{|z| \geq 1} \int_{-\infty}^{\infty}\left|h^{\prime \prime}(u)\right| d u \frac{d z}{z^{2}}=2 \int_{-\infty}^{\infty}\left|h^{\prime \prime}(u)\right| d u=2\left\|h^{\prime \prime}\right\|_{L_{1}(\mathbb{R})} .
\end{aligned}
$$

For simplicity of notation we set now

$$
a=2^{-2(\ell-1)}>0 \quad \text { and } \quad \rho(u)=\left(u^{2}+a\right)^{-t / 2} .
$$

Then $h^{\prime \prime}=\varphi_{1}^{\prime \prime} \rho+2 \varphi_{1}^{\prime} \rho^{\prime}+\varphi_{1} \rho^{\prime \prime}$. Moreover, $\rho^{\prime}(u)=-t u\left(u^{2}+a\right)^{-t / 2-1}$ and

$$
\rho^{\prime \prime}(u)=t(t+2) \frac{u^{2}}{\left(u^{2}+a\right)^{2+t / 2}}-\frac{t}{\left(u^{2}+a\right)^{2+t / 2}} .
$$

Since $1 \leq|u| \leq 3$, we have that $|\rho(u)| \leq 1$. Furthermore,

$$
2\left|\rho^{\prime}(u)\right| \leq \frac{2 t|u|}{\left(u^{2}+a\right)^{t / 2+1}} \leq \frac{2 t|u|}{|u|^{t+2}} \leq 2 t
$$


and

$$
\begin{aligned}
\left|\rho^{\prime \prime}(u)\right| & \leq t(t+2) \frac{u^{2}}{|u|^{4+t}}+\frac{t}{|u|^{4+t}} \leq t(t+2)+t \\
& =t(t+3) .
\end{aligned}
$$

We arrive at

$$
\left|h^{\prime \prime}(u)\right| \leq\left|\varphi_{1}^{\prime \prime}(u)\right|+2 t\left|\varphi_{1}^{\prime}(u)\right|+t(t+3) \varphi_{1}(u)
$$

which yields

$$
\left\|h^{\prime \prime}\left|L_{1}(\mathbb{R})\|\leq\| \varphi_{1}^{\prime \prime}\right| L_{1}(\mathbb{R})\right\|+2 t\left\|\varphi_{1}^{\prime}\left|L_{1}(\mathbb{R})\|+t(t+3)\| \varphi_{1}\right| L_{1}(\mathbb{R})\right\| .
$$

Collecting all these estimates, we get that for any $\ell \in \mathbb{N}$ we have

$$
\begin{gathered}
2^{\ell t}\left\|\mathcal{F}^{-1}\left(\frac{\varphi_{\ell}}{w^{t}}\right) \mid L_{1}(\mathbb{R})\right\| \leq 2^{t}\left(I_{1}+I_{2}\right) \leq 2^{t}\left(2\left\|\varphi_{1}\left|L_{1}(\mathbb{R})\|+2\| h^{\prime \prime}\right| L_{1}(\mathbb{R})\right\|\right) \\
\leq 2^{t+1}\left(\left\|\varphi_{1}^{\prime \prime}\left|L_{1}(\mathbb{R})\|+2 t\| \varphi_{1}^{\prime}\right| L_{1}(\mathbb{R})\right\|+(t(t+3)+1)\left\|\varphi_{1} \mid L_{1}(\mathbb{R})\right\|\right) .
\end{gathered}
$$

In the case $\ell=0$ we proceed in a similar way but now changes of variables are not needed. We have

$$
\begin{aligned}
& \left\|\mathcal{F}^{-1}\left(\frac{\varphi_{0}}{w^{t}}\right)\left|L_{1}(\mathbb{R}) \|=\int_{-\infty}^{\infty}\right| \int_{-\infty}^{\infty} \frac{\varphi_{0}(x)}{w(x)^{t}} e^{i x y} d x \mid d y\right. \\
& \quad=\int_{|y| \leq 1}\left|\int_{-\infty}^{\infty} \frac{\varphi_{0}(x)}{w(x)^{t}} e^{i x y} d x\right| d y+\int_{|y| \geq 1}\left|\int_{-\infty}^{\infty} \frac{\varphi_{0}(x)}{w(x)^{t}} e^{i x y} d x\right| d y \\
& \quad=I_{1}+I_{2} .
\end{aligned}
$$

Using the estimate $w(x) \geq 1$ we get

$$
I_{1} \leq \int_{|y| \leq 1} \int_{-\infty}^{\infty} \varphi_{0}(x) d x d y=2\left\|\varphi_{0} \mid L_{1}(\mathbb{R})\right\|
$$

and $I_{2} \leq 2\left\|h^{\prime \prime} \mid L_{1}(\mathbb{R})\right\|$, where now $h(x)=\varphi_{0}(x) / w(x)^{t}$. This time we put $\rho(x)=\left(x^{2}+1\right)^{-t / 2} \leq 1$. We obtain $\left|\rho^{\prime}(x)\right| \leq t$ and $\left|\rho^{\prime \prime}(x)\right| \leq t(t+3)$. Hence

$$
\left|h^{\prime \prime}(x)\right| \leq\left|\varphi_{0}^{\prime \prime}(x)\right|+2 t\left|\varphi_{0}^{\prime}(x)\right|+t(t+3) \varphi_{0}(x)
$$

and therefore

$\left\|\mathcal{F}^{-1}\left(\frac{\varphi_{0}}{w^{t}}\right) \mid L_{1}(\mathbb{R})\right\| \leq 2\left(\left\|\varphi_{0}^{\prime \prime}\left|L_{1}(\mathbb{R})\|+2 t\| \varphi_{0}^{\prime}\right| L_{1}(\mathbb{R})\right\|+(t(t+3)+1)\left\|\varphi_{0} \mid L_{1}(\mathbb{R})\right\|\right)$.

This establishes (4.6) and completes the proof.

Remark 4.11. The proof works (with some simplifications) also for $t=0$ where there is no lifting operator. We obtain that

$$
\left\|I_{d}: L_{1}\left(\mathbb{T}^{d}\right) \longrightarrow S_{1, \infty}^{0} B\left(\mathbb{T}^{d}\right)\right\| \leq 2^{d} C(\varphi)^{d},
$$

with

$$
C(\varphi)=\max _{j=0,1}\left(\left\|\varphi_{j}\left|L_{1}(\mathbb{R})\|+\| \varphi_{j}^{\prime \prime}\right| L_{1}(\mathbb{R})\right\|\right) .
$$


Corollary 4.12. Let $F_{d}(w)$ be given by a weight $w$ satisfying $\sum_{k \in \mathbb{Z}^{d}} w(k)^{-2}<$ $\infty$, and let $\left(\sigma_{j}\right)_{j \in \mathbb{N}}$ denote the non-increasing rearrangement of $(1 / w(k))_{k \in \mathbb{Z}^{d}}$. Then one has for all $n \in \mathbb{N}$

$$
2^{-d} C(\varphi)^{-d}\left(\sum_{j=n}^{\infty} \sigma_{j}^{2}\right)^{1 / 2} \leq a_{n}\left(I_{d}: S_{1, \infty}^{0} B\left(\mathbb{T}^{d}\right) \rightarrow F_{d}(1 / w)\right) \leq 2^{d}\left(\sum_{j=n}^{\infty} \sigma_{j}^{2}\right)^{1 / 2}
$$

where $C(\varphi)$ is given by (4.7).

Proof. The lower estimate follows from the ideal property of approximation numbers, Remark 4.11 and Theorem 2.2. The upper estimate is a consequence of Lemma 4.7.

The asymptotic behaviour of $a_{n}\left(I_{d}: S_{1, \infty}^{t} B\left(\mathbb{T}^{d}\right) \rightarrow L_{2}\left(\mathbb{T}^{d}\right)\right)$

We close this section by describing the asymptotic behaviour of approximation numbers of the embeddings from $S_{1, \infty}^{t} B\left(\mathbb{T}^{d}\right)$ into $L_{2}\left(\mathbb{T}^{d}\right)$ and the decay of the constants as the dimension goes to infinity.

Theorem 4.13. Let $d \in \mathbb{N}$ and $t>1 / 2$. Then we have

$$
c(\varphi, t)^{-d} 2^{-(t+1) d} \leq \frac{a_{n}\left(I_{d}: S_{1, \infty}^{t} B\left(\mathbb{T}^{d}\right) \rightarrow L_{2}\left(\mathbb{T}^{d}\right)\right)}{a_{n}\left(i_{d}: H_{\mathrm{mix}}^{t, 2}\left(\mathbb{T}^{d}\right) \rightarrow L_{\infty}\left(\mathbb{T}^{d}\right)\right)} \leq 2^{d}(3 / 2)^{t d}
$$

where $c(\varphi, t)$ is given by (4.5).

Proof. Step 1. (Estimate from above.) Observe that $\left(H_{\text {mix }}^{t, 2}\left(\mathbb{T}^{d}\right)\right)^{\prime}=H_{\text {mix }}^{-t, 2}\left(\mathbb{T}^{d}\right)$ with equality of norms. Using the commutative diagram

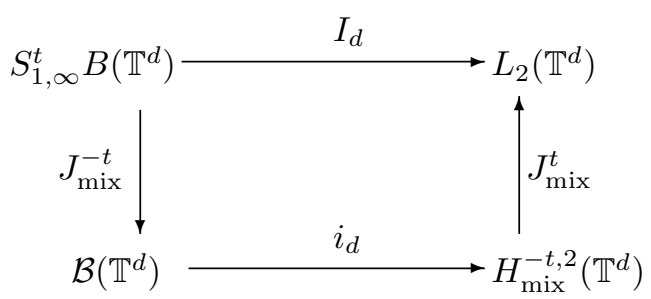

we obtain

$$
\begin{aligned}
a_{n}\left(I_{d}:\right. & \left.S_{1, \infty}^{t} B\left(\mathbb{T}^{d}\right) \rightarrow L_{2}\left(\mathbb{T}^{d}\right)\right) \leq a_{n}\left(i_{d}: \mathcal{B}\left(\mathbb{T}^{d}\right) \rightarrow H_{\text {mix }}^{-t, 2}\left(\mathbb{T}^{d}\right)\right) \\
& \times\left\|J_{\text {mix }}^{t}: H_{\text {mix }}^{-t, 2}\left(\mathbb{T}^{d}\right) \rightarrow L_{2}\left(\mathbb{T}^{d}\right)\right\|\left\|J_{\text {mix }}^{-t}: S_{1, \infty}^{t} B\left(\mathbb{T}^{d}\right) \rightarrow \mathcal{B}\left(\mathbb{T}^{d}\right)\right\| \\
& \leq 2^{d}(3 / 2)^{t d} a_{n}\left(i_{d}: \mathcal{B}\left(\mathbb{T}^{d}\right) \rightarrow H_{\text {mix }}^{-t, 2}\left(\mathbb{T}^{d}\right)\right)
\end{aligned}
$$


where we used Theorem 4.9 and that $\left\|J_{\text {mix }}^{t}: H_{\text {mix }}^{-t, 2}\left(\mathbb{T}^{d}\right) \rightarrow L_{2}\left(\mathbb{T}^{d}\right)\right\|=1$. On the other hand, by the symmetry property of approximation numbers and Theorem 2.2 , we get

$$
\begin{aligned}
a_{n}\left(i_{d}: \mathcal{B}\left(\mathbb{T}^{d}\right) \rightarrow H_{\text {mix }}^{-t, 2}\left(\mathbb{T}^{d}\right)\right) & =a_{n}\left(i_{d}: H_{\text {mix }}^{t, 2}\left(\mathbb{T}^{d}\right) \rightarrow \mathcal{A}\left(\mathbb{T}^{d}\right)\right) \\
& =a_{n}\left(i_{d}: H_{\text {mix }}^{t, 2}\left(\mathbb{T}^{d}\right) \rightarrow L_{\infty}\left(\mathbb{T}^{d}\right)\right) .
\end{aligned}
$$

Hence, the upper estimate follows.

Step 2. (Estimate from below.) Using again the symmetry property of approximation numbers, we have

$$
a_{n}\left(i_{d}: H_{\text {mix }}^{t, 2}\left(\mathbb{T}^{d}\right) \rightarrow L_{\infty}\left(\mathbb{T}^{d}\right)\right)=a_{n}\left(i_{d}: L_{1}\left(\mathbb{T}^{d}\right) \rightarrow H_{\text {mix }}^{-t, 2}\left(\mathbb{T}^{d}\right)\right)
$$

With the help of the commutative diagram

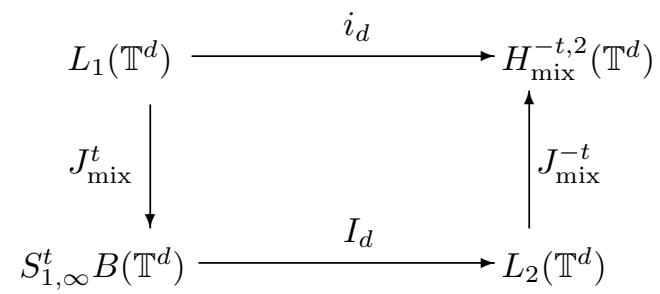

we derive

$$
\begin{gathered}
a_{n}\left(i_{d}: H_{\text {mix }}^{t, 2}\left(\mathbb{T}^{d}\right) \rightarrow L_{\infty}\left(\mathbb{T}^{d}\right)\right)=a_{n}\left(i_{d}: L_{1}\left(\mathbb{T}^{d}\right) \rightarrow H_{\text {mix }}^{-t, 2}\left(\mathbb{T}^{d}\right)\right) \\
\leq a_{n}\left(I_{d}: S_{1, \infty}^{t} B\left(\mathbb{T}^{d}\right) \rightarrow L_{2}\left(\mathbb{T}^{d}\right)\right)\left\|J_{\text {mix }}^{-t}: L_{2}\left(\mathbb{T}^{d}\right) \rightarrow H_{\text {mix }}^{-t, 2}\left(\mathbb{T}^{d}\right)\right\| \\
\quad \times\left\|J_{\text {mix }}^{t}: L_{1}\left(\mathbb{T}^{d}\right) \rightarrow S_{1, \infty}^{t} B\left(\mathbb{T}^{d}\right)\right\| \\
\leq a_{n}\left(I_{d}: S_{1, \infty}^{t} B\left(\mathbb{T}^{d}\right) \rightarrow L_{2}\left(\mathbb{T}^{d}\right)\right) c(\varphi, t)^{d} 2^{(t+1) d},
\end{gathered}
$$

where the last inequality follows by using Theorem 4.10. This gives the estimate from below.

By means of our results in [8, Corollary 4.10] this can be made explicit.

Corollary 4.14. Let $d \in \mathbb{N}$ and $t>1 / 2$. Then we have

$$
\begin{aligned}
& a_{n}\left(I_{d}: S_{1, \infty}^{t} B\left(\mathbb{T}^{d}\right) \rightarrow L_{2}\left(\mathbb{T}^{d}\right)\right) \leq 2^{d}\left(\frac{3}{2}\right)^{t d} \sqrt{\frac{2}{2 t-1}}\left[\frac{(3 \cdot \sqrt{2})^{d}}{(d-1) !}\right]^{t} \frac{(\ln n)^{(d-1) t}}{n^{t-1 / 2}} \\
& \text { if } n>\max \left(27^{d}, e^{4(d-1) t /(2 t-1)}\right), \text { and } \\
& a_{n}\left(I_{d}: S_{1, \infty}^{t} B\left(\mathbb{T}^{d}\right) \rightarrow L_{2}\left(\mathbb{T}^{d}\right)\right) \geq \\
& \qquad(\varphi, t)^{-d} 2^{-(t+1) d} \sqrt{\frac{1}{4 t-2}}\left[\frac{5}{6 d !(1+\ln \sqrt{12})^{d}}\right]^{t} \frac{(\ln (2 n))^{(d-1) t}}{n^{t-1 / 2}}
\end{aligned}
$$

if $n>\left(12 e^{2}\right)^{d}$. 
Remark 4.15. Temlyakov [34] and Romanyuk [30] (see also Romanyuk [29]) have investigated the asymptotic behavior of $a_{n}\left(I_{d}: S_{1, \infty}^{t} B\left(\mathbb{T}^{d}\right) \rightarrow L_{2}\left(\mathbb{T}^{d}\right)\right)$. But they did not consider the dependence of the constants on the dimension $d$. Corollary 4.14 also removes the technical restriction $t>1$ which appeared in [34] and [30]. Notice that $r>1 / 2$ is the optimal restriction since

$$
S_{1, \infty}^{r} B\left(\mathbb{T}^{d}\right) \hookrightarrow L_{2}\left(\mathbb{T}^{d}\right) \quad \Longleftrightarrow \quad r>1 / 2 .
$$

In fact, Temlyakov [34] and Romanyuk [30] investigated Kolmogorov numbers of the operator $I_{d}: S_{1, \infty}^{r} B\left(\mathbb{T}^{d}\right) \rightarrow L_{2}\left(\mathbb{T}^{d}\right)$, but for operators mapping a Banach space into a Hilbert space, approximation numbers and Kolmogorov numbers coincide (see [27, Proposition 11.6.2]).

Kühn, Sickel and Ullrich [20, Corollary 4.4] proved that if $t>0$ then

$$
\lim _{n \rightarrow \infty} \frac{a_{n}\left(i_{d}: H_{\mathrm{mix}}^{t, 2}\left(\mathbb{T}^{d}\right) \rightarrow L_{2}\left(\mathbb{T}^{d}\right)\right)}{n^{-t}(\ln n)^{(d-1) t}}=\left(\frac{2^{d}}{(d-1) !}\right)^{t} .
$$

Later, the present authors established in [8, Theorem 4.8] that if $t>1 / 2$ then

$$
\lim _{n \rightarrow \infty} \frac{a_{n}\left(i_{d}: H_{\mathrm{mix}}^{t, 2}\left(\mathbb{T}^{d}\right) \rightarrow L_{\infty}\left(\mathbb{T}^{d}\right)\right)}{n^{-t+1 / 2}(\ln n)^{(d-1) t}}=\frac{1}{\sqrt{2 t-1}}\left(\frac{2^{d}}{(d-1) !}\right)^{t} .
$$

For this they apply the general method to transfer $L_{2}$-estimates into $L_{\infty^{-}}$ estimates developed in [8]. These results show that there are optimal asymptotic constants for approximation numbers of $\left.i_{d}: H_{\text {mix }}^{t, 2}\left(\mathbb{T}^{d}\right) \rightarrow L_{2}\left(\mathbb{T}^{d}\right)\right)$ and $\left.i_{d}: H_{\text {mix }}^{t, 2}\left(\mathbb{T}^{d}\right) \rightarrow L_{\infty}\left(\mathbb{T}^{d}\right)\right)$ in the sense that the above limits exists. Note that the constant $\left(2^{d} /(d-1) !\right)^{t}$ decays super-exponentially as $d$ goes to infinity.

Combining Theorem 4.13 with the result above we derive the following.

Theorem 4.16. Let $d \in \mathbb{N}$ and $t>1 / 2$. Then

$$
\begin{gathered}
\limsup _{n \rightarrow \infty} \frac{a_{n}\left(I_{d}: S_{1, \infty}^{t} B\left(\mathbb{T}^{d}\right) \rightarrow L_{2}\left(\mathbb{T}^{d}\right)\right)}{n^{-t+1 / 2}(\ln n)^{(d-1) t}} \leq \frac{2^{d}}{\sqrt{2 t-1}}\left(\frac{3^{d}}{(d-1) !}\right)^{t}, \\
\liminf _{n \rightarrow \infty} \frac{a_{n}\left(I_{d}: S_{1, \infty}^{t} B\left(\mathbb{T}^{d}\right) \rightarrow L_{2}\left(\mathbb{T}^{d}\right)\right)}{n^{-t+1 / 2}(\ln n)^{(d-1) t}} \geq \frac{1}{2^{d} c(\varphi, t)^{d}((d-1) !)^{t} \sqrt{2 t-1}},
\end{gathered}
$$

where $c(\varphi, t)$ is the constant given in (4.5).

Note that the constants in the last statement

$$
\frac{2^{d}}{\sqrt{2 t-1}}\left(\frac{3^{d}}{(d-1) !}\right)^{t}, \quad \frac{1}{2^{d} c(\varphi, t)^{d}((d-1) !)^{t} \sqrt{2 t-1}}
$$

decay also super-exponentially as $d$ goes to infinity. 


\section{On the approximation of tensor products of functions}

We start this section by introducing the family of spaces $\left\{\mathcal{B}^{t}\left(\mathbb{T}^{d}\right)\right\}_{t \in \mathbb{R}}$ which are related to $\mathcal{B}\left(\mathbb{T}^{d}\right)$. As it will be clear later, the space $\mathcal{B}^{1}\left(\mathbb{T}^{d}\right)$ is of special interest because it is also connected with tensor products of functions with finite total variation and with the Sobolev space of dominating mixed smoothness based on $L_{1}\left(\mathbb{T}^{d}\right)$.

Let $t \in \mathbb{R}$. We put

$$
\mathcal{B}^{t}\left(\mathbb{T}^{d}\right)=\left\{g \in D^{\prime}\left(\mathbb{T}^{d}\right):\left\|g\left|\mathcal{B}^{t}\left(\mathbb{T}^{d}\right) \|=\sup _{k \in \mathbb{Z}^{d}}\right| \widehat{g}(k) \mid \prod_{j=1}^{d}\left(1+\left|k_{j}\right|^{2}\right)^{t / 2}<\infty\right\} .\right.
$$

Clearly, we have $J_{\text {mix }}^{t}\left(\mathcal{B}^{t}\left(\mathbb{T}^{d}\right)\right)=\mathcal{B}\left(\mathbb{T}^{d}\right)$. Even more is true. The mapping $J_{\text {mix }}^{t}$ is an isometry.

Corollary 5.1. Let $t>1 / 2$. Then one has for all $n \in \mathbb{N}$

$$
\begin{aligned}
a_{n}\left(I_{d}: \mathcal{B}^{t}\left(\mathbb{T}^{d}\right) \rightarrow L_{2}\left(\mathbb{T}^{d}\right)\right) & =a_{n}\left(i_{d}: \mathcal{B}\left(\mathbb{T}^{d}\right) \rightarrow H_{\text {mix }}^{-t, 2}\left(\mathbb{T}^{d}\right)\right) \\
& =a_{n}\left(i_{d}: H_{\text {mix }}^{t, 2}\left(\mathbb{T}^{d}\right) \rightarrow L_{\infty}\left(\mathbb{T}^{d}\right)\right) .
\end{aligned}
$$

Proof. In view of the commutative diagram

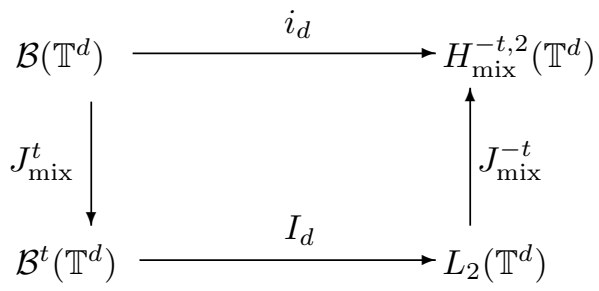

the proof of (5.1) is obvious. Now (5.2) follows from Corollary 4.5 and Theorem 2.2 .

From now on we concentrate on $t=1$. This seems to be the most interesting case. However, there are natural extensions to higher values as well.

Let $S_{1}^{1} W\left(\mathbb{T}^{d}\right)$ denote the Sobolev space of dominating mixed smoothness based on $L_{1}\left(\mathbb{T}^{d}\right)$, i.e., a function $f \in L_{1}\left(\mathbb{T}^{d}\right)$ belongs to $S_{1}^{1} W\left(\mathbb{T}^{d}\right)$ if

$$
\frac{\partial^{d} f}{\partial x_{1} \cdot \partial x_{2} \cdot \ldots \cdot \partial x_{d}} \in L_{1}\left(\mathbb{T}^{d}\right) .
$$

Then the Gagliardo-Nirenberg inequalities show that all derivatives of the form

$$
\frac{\partial^{\ell} f}{\partial x_{j_{1}} \cdot \ldots \cdot \partial x_{j_{\ell}}}, \quad \ell=1, \ldots d-1, \quad j_{1}, j_{2}, \ldots, j_{\ell} \in\{1, \ldots, d\},
$$

$j_{i} \neq j_{n}$ for all $i, n, i \neq n$, belong to $L_{1}\left(\mathbb{T}^{d}\right)$ as well. We put

$$
\left\|f\left|S_{1}^{1} W\left(\mathbb{T}^{d}\right)\|:=\| f\right| L_{1}\left(\mathbb{T}^{d}\right)\right\|+\left\|\frac{\partial^{d} f}{\partial x_{1} \partial x_{2} \ldots \partial x_{d}} \mid L_{1}\left(\mathbb{T}^{d}\right)\right\| .
$$


All derivatives have to be understood in the distributional sense. Let $k \in \mathbb{Z}^{d}$ be such that all components are different from 0 . Iterated integration by parts in the formula

$$
\hat{f}(k)=(2 \pi)^{-d} \int_{\mathbb{T}^{d}} f(y) e^{-i k y} d y
$$

yields

$$
\hat{f}(k)=\left(\prod_{j=1}^{d}\left(-i k_{j}\right)^{-1}\right)(2 \pi)^{-d} \int_{\mathbb{T}^{d}} \frac{\partial^{d} f}{\partial x_{1} \cdot \partial x_{2} \cdot \ldots \cdot \partial x_{d}}(y) e^{-i k y} d y .
$$

A similar argument can be applied if a few components of $k$ are 0. More exactly, let $k=\left(k_{1}, \ldots k_{\ell}, 0, \ldots, 0\right)$. Then we shall work with the derivative $\frac{\partial^{\ell} f}{\partial x_{1} \cdot \ldots \cdot \partial x_{\ell}}$ and argue as above. This gives

$$
S_{1}^{1} W\left(\mathbb{T}^{d}\right) \hookrightarrow \mathcal{B}^{1}\left(\mathbb{T}^{d}\right) .
$$

There is a further subspace of $\mathcal{B}^{1}\left(\mathbb{T}^{d}\right)$ which is of interest. Let $n \in \mathbb{N}$. By $\mathfrak{Z}_{n}$ we denote a partition of $[-\pi, \pi]$, i.e., a set of points

$$
-\pi \leq t_{1}<t_{2}<\ldots<t_{n+1} \leq \pi .
$$

For a function $f: \mathbb{R} \rightarrow \mathbb{C}$ we define the total variation of $f$ by

$$
\operatorname{Var} f:=\sup _{n \in \mathbb{N}} \sup _{\mathfrak{Z}_{n}} \sum_{j=1}^{n}\left|f\left(t_{j+1}\right)-f\left(t_{j}\right)\right| .
$$

Then $B V(\mathbb{T})$ is the collection of all $2 \pi$-periodic functions with finite total variation. We put

$$
\left\|f\left|B V(\mathbb{T}) \|:=\sup _{t \in \mathbb{R}}\right| f(t) \mid+\operatorname{Var} f .\right.
$$

We would like to compare the space $B V(\mathbb{T})$ with some distribution spaces like Besov spaces. This needs some care. We shall use a standard approach, see, e.g. [10, pp. 17]. Functions in $B V(\mathbb{T})$ have at most a countable set of discontinuities and at each point $t$ the left and the right limits $f(t-)$ and $f(t+)$ exist. To each function $f$ in $B V(\mathbb{T})$ we associate a function $\bar{f}$ as follows

$$
\bar{f}(t):=\frac{f(t+)+f(t-)}{2}
$$

and define

$$
\operatorname{Var}^{*} f:=\operatorname{Var} \bar{f} .
$$

Let $\operatorname{Lip}\left(1, L_{1}(\mathbb{T})\right)$ be the collection of all $f \in L_{1}(\mathbb{T})$ such that

$$
\sup _{t>0} t^{-1} \sup _{|h|<t} \int_{-\pi}^{\pi}|f(t+h)-f(t)| d t<\infty .
$$

In [10, Theorem 9.3] it is proved that a function $f \in L_{1}(\mathbb{T})$ belongs to $\operatorname{Lip}\left(1, L_{1}(\mathbb{T})\right)$ if and only if $f$ can be corrected on a set of measure zero to be a function $g \in B V(\mathbb{T})$. Moreover

$$
\sup _{t>0} t^{-1} \sup _{|h|<t} \int_{-\pi}^{\pi}|f(t+h)-f(t)| d t=\operatorname{Var}^{*} g .
$$


Since $f \in L_{1}(\mathbb{T})$ and

$$
\sup _{t>0} t^{-1} \sup _{|h|<t} \int_{-\pi}^{\pi}|f(t+2 h)-2 f(t+h)+f(t)| d t<\infty
$$

imply $f \in B_{1, \infty}^{1}(\mathbb{T})$, see [31, Theorem 3.5.4], we have the continuous embedding

$$
B V(\mathbb{T}) \hookrightarrow B_{1, \infty}^{1}(\mathbb{T}) .
$$

where $B V(\mathbb{T})$ is equipped with the norm

$$
\left\|f\left|B V(\mathbb{T}) \|^{*}:=\sup _{t \in \mathbb{R}}\right| f(t) \mid+\operatorname{Var}^{*} f .\right.
$$

Standard arguments with respect to Riemann-Steiltjes integrals yield

$$
|\hat{f}(k)| \leq \frac{1}{k} \operatorname{Var}^{*} f, \quad k \in \mathbb{Z} \backslash 0,
$$

see, e.g., [4, Prop. 4.23]. Therefore

$$
B V(\mathbb{T}) \hookrightarrow \mathcal{B}^{1}(\mathbb{T}) .
$$

Now we turn to tensor products. We put

$$
\begin{aligned}
B V(\mathbb{T}) \otimes \ldots \otimes B V(\mathbb{T}) & :=\left\{h=\sum_{j=1}^{n} f_{j, 1} \otimes f_{j, 2} \otimes \ldots \otimes f_{j, d}:\right. \\
n & \left.\in \mathbb{N}, f_{j, \ell} \in B V(\mathbb{T}), j=1, \ldots, n, \ell=1, \ldots, d\right\} .
\end{aligned}
$$

The algebraic tensor product is a vector space, and the embedding (5.4) yields the inclusion

$$
B V(\mathbb{T}) \otimes \ldots \otimes B V(\mathbb{T}) \subset \mathcal{B}^{1}\left(\mathbb{T}^{d}\right) .
$$

In order to turn the $d$-fold algebraic tensor product into a normed space we use the projective tensor norm, starting with $d=2$. Let $X$ and $Y$ be Banach spaces. Then the projective tensor norm $\gamma(\cdot, X, Y)$ is defined by

$$
\gamma(h, X, Y)=\inf \left\{\sum_{j=1}^{n}\left\|f_{j}\left|X\|\| g_{j}\right| Y\right\|: \quad f_{j} \in X, g_{j} \in Y, h=\sum_{j=1}^{n} f_{j} \otimes g_{j}\right\} .
$$

By $B V(\mathbb{T}) \widehat{\otimes} B V(\mathbb{T})$ we denote the completion of $B V(\mathbb{T}) \otimes B V(\mathbb{T})$ with respect to $\gamma$. Endowed with $\gamma$ the set $B V(\mathbb{T}) \widehat{\otimes} B V(\mathbb{T})$ becomes a Banach space. Let

$$
h=\sum_{j=1}^{n} f_{j} \otimes g_{j}, \quad f_{j}, g_{j} \in B V(\mathbb{T}) .
$$

Then

$$
\begin{aligned}
\left|\hat{h}\left(k_{1}, k_{2}\right)\right| & \leq \sum_{j=1}^{n}\left|\hat{f}_{j}\left(k_{1}\right)\right|\left|\hat{g}_{j}\left(k_{2}\right)\right| \\
& \leq\left|k_{1}\right|\left|k_{2}\right| \sum_{j=1}^{n}\left\|f_{j}\left|B V(\mathbb{T})\|\| g_{j}\right| B V(\mathbb{T}) \mid\right.
\end{aligned}
$$


which proves

$$
\left\|h\left|\mathcal{B}^{1}\left(\mathbb{T}^{2}\right)\|\leq\| h\right| B V(\mathbb{T}) \widehat{\otimes} B V(\mathbb{T})\right\|,
$$

i.e.,

$$
B V(\mathbb{T}) \widehat{\otimes} B V(\mathbb{T}) \hookrightarrow \mathcal{B}^{1}\left(\mathbb{T}^{2}\right) .
$$

We proceed by induction with respect to $d$. The space

$$
B V(\mathbb{T}) \widehat{\otimes} B V(\mathbb{T}) \widehat{\otimes} B V(\mathbb{T})
$$

is defined as tensor product of $X:=B V(\mathbb{T}) \widehat{\otimes} B V(\mathbb{T})$ with $Y:=B V(\mathbb{T})$ equipped with the norm $\gamma(\cdot, X, Y)$ and so on. We shall use the abbreviation

$$
T B V\left(\mathbb{T}^{d}\right):=B V(\mathbb{T}) \widehat{\otimes} \ldots \widehat{\otimes} B V(\mathbb{T})
$$

and call it the tensor product BV space. For the basics in theory of tensor products of Banach spaces we refer to [9] and [21]. Mathematical induction proves

$$
T B V\left(\mathbb{T}^{d}\right) \hookrightarrow \mathcal{B}^{1}\left(\mathbb{T}^{d}\right)
$$

for all $d$.

It would be of certain interest to clarify the relation of $T B V\left(\mathbb{T}^{d}\right)$ to the various other notions of multivariate variation, e.g., variation in the sense of Hardy and Krause, or in the sense of Vitali or Frechet or Tonelli, see [4, Sect. 1.4]. Whereas classically the interest is concentrated on the two-dimensional case, generalizations around the Koksma-Hlawka inequality are mainly interested in the $d$-dimensional case with $d$ large, we refer to Owen [25], Aistleitner, Dick [1] and Aistleitner et al. [2].

Corollary 5.2. Let $d \in \mathbb{N}$ and $n>\max \left(27^{d}, e^{4(d-1)}\right)$. Then there exists a linear operator $A_{n}$ of rank $<n$ such that

$$
\left\|f-A_{n} f\left|L_{2}(\mathbb{T})\|\leq\| f\right| \mathcal{B}^{1}\left(\mathbb{T}^{d}\right)\right\| \sqrt{2}\left[\frac{(3 \cdot \sqrt{2})^{d}}{(d-1) !}\right] \frac{(\ln n)^{(d-1)}}{n^{1 / 2}} .
$$

Proof. The proof is an immediate consequence of Corollary 5.1 and Corollary 4.10 in $[8]$.

Remark 5.3. From (5.3), (5.5) we conclude that tensor products $f_{1} \otimes \ldots \otimes f_{d}$ of functions $f_{i}, i=1, \ldots, d$, belonging either to $W_{1}^{1}(\mathbb{T})$ or to $B V(\mathbb{T})$ can be approximated in $L_{2}\left(\mathbb{T}^{d}\right)$ by a rate $C(d) \frac{(\ln n)^{(d-1)}}{n^{1 / 2}}$. However, most interesting is the given estimate of the constant $C(d)$, which is, as mentioned before, decaying superexponentially in $d$.

An overview about the behaviour of approximation numbers of embeddings of Sobolev or Besov spaces into $L_{p}$-spaces can be found in the monographs by Edmunds, Triebel [13] (isotropic case), Temlyakov [35], [36], Triebel [39] and Dũng, Temlyakov, Ullrich [12]. Up to now only in a few situations one knows estimates for approximation numbers of embeddings of Sobolev or Besov spaces into $L_{p}$-spaces with explicit dependence on $d$, we refer to the papers by Krieg [16], Kühn, Sickel, Ullrich [19], [20], Kühn, Mayer, Ullrich [18], the present authors [8], and Mieth [23]. Recently Bachmayr et al. [5] have investigated the approximation of tensor products of functions belonging to $W_{\infty}^{t}(0,1)$ in the 
$L_{\infty}$-norm, where $t$ is a given natural number, see also Novak, Rudolf [24] and Krieg, Rudolf [17]. They have been able to show that there is an algorithm which uses $n$ function values and approximates with a rate $n^{-t}$. The price they have to pay is a huge constant depending on $d$ in the related inequality. The known rate of $a_{n}\left(I_{d}: W_{m i x}^{t}\left((0,1)^{d}\right) \rightarrow L_{\infty}\left((0,1)^{d}\right)\right.$ is given by $\frac{(\ln n)^{(t+1)(d-1)}}{n^{t}}$, see [35, IV.5]. However, here the dependence of the hidden constants on $d$ is not known.

We conclude the paper with a number of related open questions.

- Is there an analog of the result of Bachmayr et al. [5] for the pair $\left(\mathcal{B}^{1}\left(\mathbb{T}^{d}\right), L_{2}\left(\mathbb{T}^{d}\right)\right)$ or even simpler, can the inequality (5.6) be improved by concentrating on tensor products of functions from $B V(\mathbb{T})$ instead of $\mathcal{B}^{1}\left(\mathbb{T}^{d}\right) ?$

- What about $n \leq \max \left(27^{d}, e^{4(d-1)}\right)$ ? From the practical point of view this is the more important range. First results for the so-called preasymptotic range have been obtained in [6], [32], [19], [20], [18] and [16]. However, up to now related results for $a_{n}\left(I_{d}: H_{m i x}^{t}\left(\mathbb{T}^{d}\right) \rightarrow L_{\infty}\left(\mathbb{T}^{d}\right)\right)$ are not known.

- Beside approximation numbers there are other related quantities, like Kolmogorov $\left(d_{n}(T)\right)$, Gelfand $\left(c_{n}(T)\right)$, Weyl $\left(x_{n}(T)\right)$ or Bernstein numbers. Some relationships between them are known. For example, $a_{n}(T)=d_{n}(T)$ for operators $T$ mapping into a Hilbert space and $a_{n}(T)=c_{n}(T)=x_{n}(T)$ for operators starting from a Hilbert space. Of course, it would be desirable to have analogous results for these numbers, too. But this would require a different proof technique, so we leave this as an open problem.

\section{References}

[1] C. Aistleitner and J. Dick, Functions of bounded variation, signed measures and a general Koksma-Hlawka inequality, Acta Arithmetica 167 (2015), 143-171.

[2] C. Aistleitner, F. Pausinger, A.M. Svane and R.F. Tichy, On functions of bounded variation, Math. Proc. Camb. Phil. Soc. 162 (2017), 405-418.

[3] T.I. Amanov, Spaces of differentiable functions with dominant mixed derivative, Nauka, Alma-Ata, 1976.

[4] J. Appell, J.Banaz and N.J. Merentez Diaz, Bounded variation and around, De Gruyter, Berlin, 2013.

[5] M. Bachmayr, W. Dahmen, R.A. DeVore and L. Grasedyck, Approximation of high-dimensional rank one tensors, Constr. Approx. 39 (2014), 385-395.

[6] H.-J. Bungartz and M. Griebel, Sparse grids, Acta Numer. 13 (2004), 147269.

[7] B. Carl and I. Stephani, Entropy, compactness and the approximation of operators, Cambridge Univ. Press, Cambridge, 1990.

[8] F. Cobos, T. Kühn, and W. Sickel, Optimal approximation of multivariate periodic Sobolev functions in the sup-norm, J. Funct. Anal. 270 (2016) 4196-4212. 
[9] A. Defant and K. Floret, Tensor norms and operator ideals, North Holland, Amsterdam, 1993.

[10] R.A. DeVore and G.G. Lorentz, Constructive Approximation, Springer, Berlin, 1993.

[11] D. Dũng and T. Ullrich, $N$-Widths and $\varepsilon$-dimensions for high-dimensional approximations, Found. Comp. Math 13 (2013), 965-1003.

[12] D. Dũng, V.N. Temlyakov, T. Ullrich, Hyperbolic Cross Approximation, Advanced Courses in Mathematics - CRM Barcelona, Springer, Barcelona, 2018.

[13] D.E. Edmunds and H. Triebel, Function spaces, entropy numbers, differential operators, Cambridge Tracts in Mathematics 120, Cambridge University Press, Cambridge, 1996.

[14] R. E. Edwards, Fourier series. A modern introduction, Springer, New York, 1979.

[15] C.V. Hutton, On the approximation numbers of an operator and its adjoint, Math. Ann. 210 (1974), 277-280.

[16] D. Krieg, Tensor sequences and the approximation of tensor product operators, J. Complexity 44 (2018), 30-51.

[17] D. Krieg and D. Rudolf, Recovery algorithms for high-dimensional rank one tensors, J. Approx. Theory 237 (2019), 17-29.

[18] T. Kühn, S. Mayer, and T. Ullrich, Counting via entropy - new preasymptotics for the approximation numbers of Sobolev embeddings, Siam J. Numer. Anal 54(6)(2016), 3625-3647.

[19] T. Kühn, W. Sickel and T. Ullrich, Approximation numbers of Sobolev embeddings - Sharp constants and tractability, J. Complexity 30 (2014), 95-116.

[20] T. Kühn, W. Sickel and T. Ullrich, Approximation of mixed order Sobolev functions on the d-torus - Asymptotics, preasymptotics and d-dependence, Constr. Approx. 42 (2015), 353-398.

[21] W.A. Light and E.W. Cheney, Approximation theory in tensor product spaces, Lecture Notes in Math. 1169, Springer, Berlin, 1985.

[22] P.I. Lizorkin and S.M. Nikol'skij, Function spaces of mixed smoothness from the decomposition point of view (Russian), Trudy Mat. Inst. Steklova $\mathbf{1 8 7}$ (1989), 143-161, translated in Proc. Steklov Inst. Math. (1990), no.3, 163184.

[23] T. Mieth, Sharp estimates for approximation numbers of non-periodic Sobolev spaces, arXiv:1811.01576 [math.FA], to appear in J. Complexity

[24] E. Novak and D. Rudolf, Tractability of the approximation of highdimensional rank one tensors, Constr. Approx. 43 (2016), 1-13. 
[25] A.B. Owen, Multi-dimensional varaiton for quasi-Monte Carlo. Contemporary multivariate analysis and design of experiments, 49-74, Ser. Biostat. 2, World Sientific Publ. Co. Pte. Ltd., Hackensack, NJ, 2006.

[26] J. Peetre, New thoughts on Besov spaces, Duke Univ. Math. Series, Durham, 1976.

[27] A. Pietsch, Operator Ideals, North-Holland, Amsterdam, 1980.

[28] A. Pietsch, Eigenvalues and s-numbers, Cambridge University Press, Cambridge, 1987.

[29] A.S. Romanyuk, Linear widths of the Besov classes of periodic functions of many variables. I, Ukr. Math. J. 53 (1) (2001), 744-761.

[30] A.S. Romanyuk, Kolmogorov and trigonometric widths of the Besov classes $B_{p, \theta}^{r}$ of multivariate periodic functions, Mat. Sbornik 197 (1) (2006), 71-96.

[31] H.-J. Schmeisser, H. Triebel, Topics in Fourier analysis and function spaces, Geest \& Portig, Leipzig, 1987 and Wiley, Chichester, 1987.

[32] Ch. Schwab, E. Süli and R.A. Todor, Sparse finite element approximation of high-dimensional transport-dominated diffusion problems, ESAIM: Mathematical Modelling and Numerical Analysis 42 (05) (2008), 777-819.

[33] W. Sickel and H. Triebel, Hölder inequalities and sharp embeddings in function spaces of $B_{p, q}^{s}$ and $F_{p, q}^{s}$ type, Z. Anal. Anwendungen 14 (1995), 105-140.

[34] V.N. Temlyakov, Approximation of periodic functions of several variables by trigonometric polynomials and widths of some classes of functions, Math. USSR Izvestiya 27 (1986), 285-322.

[35] V.N. Temlyakov, Approximation of periodic functions, Nova Science, New York, 1993.

[36] V.N. Temlyakov, Multivariate approximation, Cambridge Univ. Press, Cambridge, 2017.

[37] H. Triebel, Fourier analysis and function spaces, Teubner-Texte Math. 7, Teubner, Leipzig, 1977.

[38] H. Triebel, Theory of function spaces, Geest \& Portig, Leipzig, 1983 and Birkhäuser, Basel, 1983.

[39] H. Triebel, Bases in function spaces, sampling, discrepancy, numerical integration, EMS Tracts in Mathematics 11, European Mathematical Society, Zürich, 2010.

[40] H. Yserentant, Regularity and Approximability of Electronic Wave Functions, Springer, Berlin, 2010. 